\title{
Competencia Comunicativa en la Formación Inicial Actual del Profesor de Matemática en Chile
}

\author{
Claudia Vargas-Díaz y Hank Apablaza \\ Departamento de Matemática y Ciencia de la Computación, Universidad de Santiago de Chile, Santiago - Chile \\ (e-mail: claudia.vargas.d@usach.cl; hank.apablaza@usach.cl)
}

Recibido Jul. 10, 2018; Aceptado Sep. 25, 2018; Versión final Nov. 30, 2018, Publicado Jun. 2019

\begin{abstract}
Resumen
El artículo discute y analiza la situación actual en el desarrollo de la competencia comunicativa en la formación del profesor de matemática en Chile. Se revisaron los planes de formación de carreras de pedagogía en educación matemática de las universidades del Consejo de Rectores de Universidades de Chile disponibles en línea. Además, se confeccionó un cuestionario para explorar las opiniones de directores de dichos programas del profesor de matemática en Chile acerca de la formación en dichas competencias comunicativas. Los resultados obtenidos muestran algunas concepciones comunes. Por ejemplo, que aún se considera que la competencia comunicativa del profesor de matemática está relacionada más bien con elementos de la comunicación oral y escrita, o sea como una habilidad profesional de índole general y no específica del profesor de matemática. Como conclusión, se establece que en Chile aún se debe instalar el desarrollo de competencia comunicativa para enseñar matemática en la formación inicial del futuro profesor.
\end{abstract}

\section{Communicative Competence in Current Mathematics Teachers' Formation in Chile}

\begin{abstract}
In this paper the current state in the development of communicative competence in mathematics teachers' formation in Chile is discussed and analyzed. The online descriptions of the mathematic teachers' formation programs for the universities that are part of the Rector's Council of Chilean Universities were obtained. A questionnaire was designed to explore the opinions of the directors of these programs about the formation in communicative competences. The results show that there are some common erroneous conceptions. For instance, that the communicative competence of mathematics teachers is still considered part of oral and written communication, that is to say: as a general ability instead of an ability specialized for teaching mathematics. It is concluded that in Chile it is still necessary to introduce the development of communicative competences in mathematics teaching during the initial formation of future teachers.
\end{abstract}

Keywords: communicative competence; teachers' formation programs; mathematics teachers; professional development 


\section{INTRODUCCIÓN}

Aprender a comunicar matemática debería ser parte de la trayectoria formativa del futuro profesor de matemática en un programa de formación de docentes de todos los países. La investigación en la formación por competencia profesionales del profesor (Fernández y Cuadrado, 2010) ha ido incrementando y la competencia de comunicar eficazmente las matemática comienza a ser importante (Giménez y Vargas, 2012), considerando que también se estudia la interacción en el aula (Villalta, 2009), (Villalta et al, 2011), (Villalta y Martinic, 2013) para precisar el tipo de intercambios que en ella se producen evidenciando el papel del profesor. Por ello, es preciso reconocer si se está desarrollando en los procesos formativos mencionados, además de detectar fortalezas y debilidades de los egresados en el ejercicio de la profesión docente en matemática. En lo que concierne a la cognición, se ha abordado un tema relacionado como lo es el lenguaje matemático desde la perspectiva del habla de los niños en el aula y de los trabajos escritos para los alumnos, es decir, en el contexto hablado y escrito (Pimm,1987). En ese momento, fue importante explorar aspectos del lenguaje matemático en el aula, de profesor a alumno y también entre los alumnos, tanto escrito como oral. Actualmente, el cómo aprender a enseñar matemática es una pregunta que ha llevado a examinar al profesor de matemática en su rol de mediador entre el alumno y el conocimiento matemático (Planas, 2012), donde no tiene sentido mirar al profesor y al conocimiento como factores distintos e independientes. Lo anterior, dado que la mediación se da a través del lenguaje que los relaciona a ambos, profesor y conocimiento matemático, y hacia el alumno (Ibañez, 2007).

En las dos últimas décadas se ha incorporado la noción de competencia en la formación del profesor (Gairín, 2011) (Lomas, 2003) y también se ha incrementado la investigación en este campo intentando responder a preguntas como: ¿cuál es la situación actual en los programas de educación matemática en términos de competencia de enseñanza en el aula? (Gokalp, 2015), (Galvis, 2007). Así, la formación del profesor de matemática se ha ido adaptando a las nuevas miradas por competencia profesionales (Giménez et al, 2011) abriendo paso a las competencia comunicativa como una competencia específica a desarrollar. Esta se considera como una competencia transversal en la formación del profesor de matemática (Giménez y Vargas, 2012) en la cual se considera que se comunica cuando se reconoce al otro y se contrasta con él. Es decir, cuando en la comunicación que se produce en el aula existe una reciprocidad, un sí y solo sí de los participantes, entre los alumnos y el docente. Para la enseñanza de las matemática, y pensado para alumnos de cualquier etapa educativa, se caracterizó la noción de competencia matemática como la habilidad de entender, juzgar, hacer y usar la matemática en una variedad de situaciones y contextos intra y extramatemáticos, en los cuales las matemática juegan o podrían jugar un rol (Niss, 2002). En ese contexto se sitúa "comunicar en, con y acerca de las matemática" como una de las ocho competencia diferentes que describen las acciones, habilidades y destrezas en la actividad matemática. Destacamos que en la comunicación de la matemática se quiere dar importancia a: 1) entender lo que otros escriben, en cuanto a textos orales o visuales, en una variedad de registros lingüísticos acerca de materias que tienen un contenido matemático; y 2) expresarse en diferentes niveles de precisión teórica y técnica de forma oral, visual y escrita acerca de tales materias. Con la contextualización anterior se sienta una base para hablar de competencia matemática, la cual involucra la comunicación como foco de interés en esta investigación. De modo la competencia comunicativa matemática se define como la capacidad social comunicativa de enseñar matemática, la cual se desarrolla cuando el profesor reflexiona acerca de la importancia de la comunicación para enseñar a un grupo de alumnos interesados en aprender matemática (Vargas, 2013).

Con respecto a la formación del profesor, aunque se ha considerado el establecimiento de modelos de competencia profesionales, se han detectado la existencia de debilidades en este aspecto, así como las insuficiencias que se reflejan en planes de estudio en relación con el tratamiento de competencia comunicativa (Domingo et al, 2010). Así mismo, se visualiza un abandono en el tratamiento de la comunicación en la etapa de formación de maestros, aún cuando las consecuencias aparecerán luego en las interacciones reales del futuro profesor con sus alumnos en aula (García, 2008). En un sentido más amplio, en la formación del profesor de Chile también se ha vislumbrado la necesidad de incorporar las competencia comunicativa en el curriculum de formación del profesor (Rodríguez, 2000). Desde la investigación en formación Inicial del profesor de matemática, usando el método de observación de pares en las prácticas profesionales, se ha podido establecer que el futuro profesor presenta deficiencia en aspectos comunicativos. Precisamente estas falencias les ocasionan problemas para cumplir con este aspecto de la profesión docente (Gokalp, 2015).

En este artículo se reportan parte de los resultados del proyecto de caracterización y desarrollo de competencia comunicativa en la formación del profesor de matemática en el objetivo específico de analizar la situación actual de la formación del profesor de matemática en relación a las competencias comunicativas. A través del estudio se pudo establecer, de manera preliminar, la situación actual de la formación del profesor de matemática en relación a las competencia comunicativa en Chile en base a la información que proporcionan en internet los programas de formación del profesor de matemática y los resultados de una encuesta aplicada los jefes de carrera de cada programa participante. Estos jefes de carrera son el equivalente a director de programa o coordinador del programa de formación. 


\section{METODOLOGIA}

Hasta el año 2006 organismos externos realizaban una revisión de las carreras y otorgaban un período de acreditación para certificar la calidad de las universidades. En la actualidad, solamente puede otorgar la acreditación la Comisión Nacional de Acreditación de Chile (CNA). Como elementos del estudio se ha considerado la revisión de las carreras de pedagogía en matemática acreditadas del país y que formen parte de universidades pertenecientes al Consejo de Rectores de Universidades Chilenas (CRUCH). De este modo, se trata de programas de larga trayectoria y tradición reconocida, con titulados que se encuentran ejerciendo en el sistema escolar público y/o privado. Por otro lado, existe un consenso nacional, en el marco de la ley 20.903, e internacional, en proyectos destinados a generar políticas públicas, (Toledo y Wittemberg, 2015) para mejorar los resultados de los estudiantes, el cual indica la importancia de elevar la calidad de los docentes. Las instituciones de educación superior que están sujetas a abordar dichas políticas públicas en Chile son las universidades del Consejo de Rectores de Chile. Dentro de los indicadores de calidad de los docentes, se hace referencia al conocimiento pedagógico y, en particular en nuestra investigación, al conocimiento didáctico y matemático (Pavié, 2011) que permita generar dinámicas de enseñanza y aprendizajes efectivas para los estudiantes; monitorear y evaluar la comprensión de los aprendizajes de una clase y/o de una actividad, y tomar decisiones respecto de su quehacer pedagógico.

La formación del profesor de matemática en Chile se sustenta en un modelo híbrido donde el futuro profesor obtiene una formación en tres ámbitos básicos: cursos de matemática, de pedagogía y de didáctica. Específicamente, se observaron los perfiles de egreso de las carreras de pedagogía en matemática con las características antes mencionadas, los cuales se encuentran disponibles en internet en páginas oficiales de cada universidad. Esta información se ha considerado orientativa de lo que podría estar ocurriendo en cada programa en el tema de interés de esta investigación. De esta manera, en el marco del proyecto, primeramente, se intenta dar respuesta a una de las interrogantes principales relacionadas, a saber, ¿cómo se trata la comunicación del contenido matemático en la formación del profesor de matemática? Claramente, toda la información que no se encuentra explicitada en los datos disponibles en internet, algunos conceptos o acciones concretas, podrían haberse obviado involuntariamente en los medios digitales, por lo que la información se considera orientativa. Para indagar en más detalles sobre las carreras se realizó la encuesta online a los jefes de carrera de las universidades del $\mathrm{CRUCH}$ bajo la premisa de que, como encargados de programa, conocen mejor y poseen más información de todos los aspectos de la carrera que dirigen. Esto implicaría que están suficientemente familiarizados con las preguntas formuladas y podrían dar respuesta.

Para obtener información más precisa, se confeccionó un cuestionario de respuesta abierta (Hernández, 2014), a través correo electrónico, con el fin de recoger información más puntual sobre las carreras en el aspecto de la comunicación de la matemática. De este modo, para poder contar con detalles más específicos en cuanto a la formación del profesor de matemática en Chile, se consultó a los jefes de carrera si en las respectivas carreras se trabaja el desarrollo de competencia comunicativa en algunas asignaturas o en forma transversal. Para realizar tal actividad, se extendió la invitación a participar a todos los jefes de carreras de las universidades del $\mathrm{CRUCH}$, explicándoles debidamente la importancia del estudio y el objetivo del proyecto, cumpliendo con los lineamientos éticos del consentimiento informado de la institución patrocinadora del proyecto de investigación. Como la consulta se realizó por correo electrónico, se usó como soporte el servicio Google Forms. Por este medio se explicaron los alcances de la investigación y se solicitó el consentimiento informado, obteniendo respuesta desde siete instituciones. Cabe señalar que las restantes instituciones, a la fecha de someter a revisión este artículo, no han respondido. El cuestionario constó de cuatro preguntas y se muestra en la Tabla 1.

Tabla 1. Preguntas del Cuestionario a Jefes de Carrera.

\begin{tabular}{|l|l|}
\hline$N^{\circ}$ & Pregunta \\
\hline 1 & $\begin{array}{l}\text { Dada su experiencia, ¿Qué importancia tiene el desarrollo de habilidades comunicativa en la Formación de } \\
\text { Profesores de matemática? Argumente su respuesta y describa cuáles habilidades considera que el profesor de } \\
\text { matemática debe desarrollar en el aspecto comunicativo }\end{array}$ \\
\hline 2 & $\begin{array}{l}\text { En el proceso formativo de Profesores de matemática de su institución, ¿se abordan las habilidades comunicativa? } \\
\text { Si/No }\end{array}$ \\
\hline 3 & $\begin{array}{l}\text { De acuerdo al plan de estudio que Ud. dirige, ¿qué asignaturas desarrollan habilidades para comunicar la } \\
\text { matemática? Argumente su respuesta. }\end{array}$ \\
\hline 4 & $\begin{array}{l}\text { En la eventualidad de un rediseño curricular, ¿consideraría al menos una asignatura que desarrolle habilidades } \\
\text { comunicativa en los estudiantes de su carrera? Argumente su respuesta }\end{array}$ \\
\hline
\end{tabular}


La validación del cuestionario se realizó vía juicio experto para valorar la comprensión de las preguntas y la correcta redacción. Se consideraron como expertos los académicos universitarios, entre ellos, jefes de carreras diferentes, con trayectoria en el tema del cuestionario, y reconocidas por otros como expertos cualificados, de modo tal que pudieron afirmar si el contenido de las preguntas del instrumento resultaba adecuado (Carrión et al, 2015). Cada participante tuvo la oportunidad de aportar con observaciones que llevaron al cuestionario final. En el cuestionario, el concepto de competencia comunicativa matemática no se definió previamente a los participantes para evitar el sesgo de las respuestas y, de ese modo, adulterar inadecuadamente las respuestas. Para el análisis de las respuestas se utilizó análisis de contenido categórico para la clasificación de la respuesta escrita (Leiblich et al, 1998) y a través de este análisis se pudo sentar una base para comprender posicionamientos en las opiniones de cada participante (Stemler, 2001).

\section{RESULTADOS Y DISCUSIÓN}

Los resultados indican que en algunas de las carreras se considera la comunicación de la matemática como una de las competencia del perfil de egreso (Tabla 2). De acuerdo a lo extraído de lo expuesto en Internet (al momento de la investigación solamente cuatro universidades exponen públicamente la información), en los casos en que se expone la competencia de comunicar, esta es considerada como una competencia profesional en el sentido de saber comunicar ideas, en general en la lengua nacional o extranjera, y en términos de comunicar matemática está referida al conocimiento de la disciplina.

Tabla 2. Información sobre perfil de egreso en relación a comunicación de la matemática y comunicación profesional. Fuente: Información de carreras disponible en páginas web de universidades.

\begin{tabular}{|c|c|c|}
\hline $\begin{array}{c}\text { Universidad/ } \\
\text { Carrera }\end{array}$ & Comunicación de la Matemática & Comunicación profesional \\
\hline $\begin{array}{l}\text { Universidad de } \\
\text { Santiago de Chile } \\
\text { Licenciatura en } \\
\text { Educación } \\
\text { Matemática }\end{array}$ & $\begin{array}{l}\text { Usar un lenguaje disciplinario para describir, } \\
\text { ejemplificar, interpretar, relacionar, explicar } \\
\text { y/o fundamentar y comunicar términos, } \\
\text { principios, conceptos, objetos, reglas, } \\
\text { razonamientos, procedimientos, desarrollos } \\
\text { y soluciones. }\end{array}$ & \\
\hline $\begin{array}{l}\text { Universidad de } \\
\text { Tarapacá } \\
\text { Pedagogía en } \\
\text { Física y } \\
\text { Matemática }\end{array}$ & & $\begin{array}{l}\text { Aplicará su saber creativo y científico con criterio } \\
\text { reflexivo, tendrá mayores capacidades para } \\
\text { aprender a aprender, comunicarse y adaptarse al } \\
\text { cambio, incorporando avances tecnológicos e } \\
\text { informáticos. }\end{array}$ \\
\hline $\begin{array}{l}\text { Pontificia } \\
\text { Universidad } \\
\text { Católica de } \\
\text { Valparaíso } \\
\text { Pedagogía en } \\
\text { Matemática }\end{array}$ & $\begin{array}{l}\text { Desarrolla demostraciones matemática, las } \\
\text { que construye y elabora mediante } \\
\text { argumentaciones en las que se identifican } \\
\text { claramente las hipótesis y las conclusiones, } \\
\text { para fundamentar, expresar y comunicar } \\
\text { correctamente ideas matemática utilizando } \\
\text { el método y el lenguaje propios de la } \\
\text { disciplina. }\end{array}$ & $\begin{array}{l}\text { Utiliza correctamente idioma inglés tanto en la } \\
\text { comunicación oral como escrita, y asimismo sabe } \\
\text { interpretarlo y comprenderlo en los diferentes } \\
\text { contextos de su actividad profesional. }\end{array}$ \\
\hline $\begin{array}{l}\text { Universidad de } \\
\text { Antofagasta } \\
\text { Pedagogía en } \\
\text { Matemática }\end{array}$ & & $\begin{array}{l}\text { En suma, un profesional con capacidades } \\
\text { matemática suficientes y necesarias para ser un } \\
\text { buen diseñador, gestionador y evaluador de } \\
\text { actividades de aprendizaje de la Matemática, } \\
\text { investigador de sus propios procesos de } \\
\text { desempeño, utilizando sus capacidades de } \\
\text { comunicación y de trabajo en equipo para contribuir } \\
\text { al desarrollo sustentable, ejerciendo } \\
\text { preferentemente en contextos de diversidad y } \\
\text { multiculturalidad. }\end{array}$ \\
\hline
\end{tabular}

Se organizaron las respuestas de los siete jefes de carrera, los cuales fueron denominados A; B; C; D; E; F y G; y que corresponden a los que colaboraron respondiendo las preguntas. No necesariamente corresponden a las carreras que consideran o mencionan la comunicación como uno de sus elementos del perfil de egreso (Tabla 2). Sobre la pregunta 1, Dada su experiencia, ¿Qué importancia tiene el desarrollo de habilidades comunicativa en la Formación de Profesores de matemática? Argumente su respuesta y describa cuáles habilidades considera que el profesor de matemática debe desarrollar en el aspecto comunicativo, se obtuvieron las respuestas que a continuación se señalan.

"Para cualquier profesor es de suma importancia, para uno de matemática más aun cuando tiene que usar lenguaje común para expresar conceptos de lenguaje matemático. Manejo de vocabulario, expresión escrita y expresión oral fluida." Jefe de carrera A. 
"Las expresiones que debe desarrollar son la expresión oral y escrita, la empatía y la tolerancia que ayudan a la buena comunicación". Jefe de carrera B.

"El desarrollo de las habilidades comunicativa en lengua materna es fundamental en un profesor en un profesor. En particular en el profesor de matemática, además debe desarrollarse el lenguaje matemático (lenguaje universal). Este desarrollo es necesario para su formación de futuro profesional, su relación con otros profesionales y para propender al desarrollo de estas habilidades en sus estudiantes. Las habilidades comunicativa que debe desarrollar el profesor de matemática son: el lenguaje oral (como lo es utilizar un lenguaje adecuado, directo, modulación, inflexiones de voz,...); lenguaje escrito (redacción, ortografía, claridad en escritura de letras, palabras y números); el escuchar (empatía, uso de lenguaje no verbal, como lo es el mirar a la persona)" Jefe de carrera C.

"Debiese ser muy importante, ya que es una de las competencia básicas que fomenta nuestra universidad. Los programas de formación docente consideran al menos una asignatura. En el casi de nuestra carrera, esta competencia, además de la asignatura, está diseminada en el resto de las asignaturas que forman parte del plan de estudio." Jefe de carrera D.

"Muy importante porque debe enseñar a jóvenes y mostrarles lo entretenido e interesante que es la matemática, y la utilidad que tiene" Jefe de carrera $\mathrm{E}$.

"Es importante, no solo para el profesor de matemática. La entrega de la disciplina debe ser clara y que llegue a los alumnos, por lo tanto se espera que el profesor tenga habilidades de comunicación" Jefe de carrera F.

"Son fundamentales ya que el profesor es un mediador entre el conocimiento matemático y sus estudiantes. Sus habilidades comunicativa deben estar relacionadas con el compartir significados para lo que debe tener, entre otras, habilidades para seleccionar y construir mensajes matemáticos con sentido para los estudiantes; resignificar y aplicar distintos registros de la matemática en forma directa o a través de diferentes recursos de aprendizajes, aplicar estrategias comunicativa persuasivas que afecten positivamente la motivación de logros de los estudiantes para abordar la complejidad matemática y la confianza de ellos mismos para aprender matemática y para generar climas sociales positivo de aprendizajes que favorecen el crecimiento personal con integración social." Jefe de carrera G.

En general, se puede interpretar que los jefes de carrera distinguen dos tipos de comunicación en coherencia a lo visualizado en los perfiles de egreso: a) la comunicación de la matemática y b) la comunicación profesional relativa a expresión oral y escrita. En particular, dan más importancia al manejo de habilidades profesionales de la comunicación, es decir, aquellas habilidades que todo profesional debería poseer en cuanto a expresión oral y escrita. En el caso específico del jefe de carrera G, encontramos unas definiciones generales relacionadas con la persuasión. Todos los jefes de carrera coinciden que el desarrollo de habilidades comunicativa en el profesor de matemática es muy importante, pues mediante el lenguaje, matemático y natural, el profesor puede comunicar la disciplina a sus alumnos, mejorar el clima de aula, incentivar el interés por la disciplina, lo cual les permite, además, desarrollarse profesionalmente como profesor. Para la pregunta 2, de respuesta cerrada: En el proceso formativo del profesor de matemática de su institución, ¿se abordan las habilidades comunicativa?, la respuesta general fue categóricamente Sí, lo que nos confirma que se consideran como habilidades comunicativa las relacionadas con destrezas en el sentido de expresión oral y escrito, no explícitamente en el sentido matemático. No obstante, hubo un NO que se podría interpretar como la excepción. Con respecto a la pregunta 3: De acuerdo al plan de estudio que Ud. dirige, ¿qué asignaturas desarrollan habilidades para comunicar la matemática? Argumente su respuesta, obtuvimos lo que a continuación se señala:

"Como generales, teoría de la comunicación y habilidades de comunicación; como específica, los talleres pedagógicos y talleres curriculares por eje de la matemática. En cada asignatura de la especialidad se les exige exponer y explicar conceptos" Jefe de carrera A.

"Hay ya una asignatura que es Comunicación Efectiva, lo importante son las reuniones periódicas con el cuerpo docente para tener criterios comunes respecto del desarrollo de estas habilidades" Jefe de carrera B.

"La asignatura competencia comunicativa en específico, y en todas las del área pedagógica-profesional. No obstante a lo anterior, también en las asignaturas de didáctica específica y talleres del área de práctica" Jefe de carrera D.

"Los cursos con conocimiento pedagógico del contenido y las didácticas y las prácticas, donde se trabaja cómo enseñar la matemática y donde además se aprende a investigar en problemas de la matemática." Jefe de carrera E. 
"En algunas asignaturas se hacen disertaciones, como ser en las didácticas, en las prácticas y en alguna otra dependiendo de los programas" Jefe de carrera F.

"Aunque esta habilidad debería ser desarrollada transversalmente en el plan de estudios hay varias de ellas que contribuyen en forma especial desde el primer semestre. A modo ilustrativo, en el curso de introducción de la pedagogía se abordan varios aspectos específicos y se consideran obstáculos personales y ambientales de los estudiantes. Aun cuando en varias de ellas, de las diferentes componentes del plan de estudios, se usan códigos elaborados le proporcionan a los estudiante experiencias que fortalecen su formas de comunicación hay en varias en educación matemática que proporcionan en forma vicaria o experiencial habilidades comunicativa verbales, no verbal y escrita." Jefe de carrera G.

El jefe de carrera denominado C, considera que todas las asignaturas contribuyen al logro de estas habilidades, citando algunas de las asignaturas que, por razones de guardar el anonimato comprometido, no citaremos textualmente (se omiten los nombres para evitar que se pueda deducir la fuente). Así, se puede comentar que se explaya sobre asignaturas y sus actividades específicas, tales como disertaciones, la práctica, entre otras, y se menciona que se evalúan clases simuladas. También se hace hincapié en la relación con otros profesionales de los centros educativos donde se involucra el uso de un lenguaje adecuado. Por otra parte, se enfatiza que el trabajo de estas habilidades se comienza de forma temprana, incluso en la introducción a la pedagogía en niveles bastante prematuros de la formación inicial. En suma, los jefes de carreras participantes no informan si el plan de estudios de su institución contempla asignaturas específicas que aborden, de forma determinado, el tema de la transmisión de conocimiento matemático, por lo que no es claro que se estén trabajando estas competencias en el área de matemática. La información brindada queda en el ámbito de la comunicación profesional en general. No mencionan una asignatura que apunte a desarrollar estrategias de comunicación de la matemática y, más bien lo que se desarrolla, se encuentra diseminado en otras asignaturas.

Por otro lado, con respecto a las asignaturas de los planes de formación del profesor de matemática, se puede interpretar que los jefes de carrera afirman que las habilidades para comunicar la matemática se desarrollan transversalmente a lo largo de la carrera. Sin embargo, también mencionan que estas competencia se desarrollan, actualmente, en asignaturas que consideran elementos tales como: Metodología de la Enseñanza de la Matemática; Orientación Educacional; Prácticas Docentes; Comunicación Efectiva; Didáctica de la Matemática; Competencia Comunicativa (Estrategias de Comunicación); Taller de Comunicación; Lenguaje Matemático; Cursos de conocimiento pedagógico; Introducción a la Pedagogía en Matemática y Computación; Teoría de la Comunicación; Habilidades de Comunicación.

En relación a la pregunta 4 y final, En la eventualidad de un rediseño curricular, ¿consideraría al menos una asignatura que desarrolle habilidades comunicativas en los estudiantes de su carrera? Argumente su respuesta, se obtuvieron las siguientes afirmaciones:

"Ya las tenemos, la competencia de comunicación es una de las competencia de nuestro plan de estudios." Jefe de carrera A.

"Hay ya una asignatura que es Comunicación Efectiva, lo importante son las reuniones periódicas con el cuerpo docente para tener criterios comunes respecto del desarrollo de estas habilidades." Jefe de carrera B.

"Si, En la propuesta de perfil para el egresado de pedagogía de nuestra institución (las carreras se encuentran en proceso de renovación curricular), se han incluido capacidades en esta dirección, como lo son: comunicación oral y escrita de manera efectiva, coherente y correcta en diversos contextos profesionales; capacidad de comprensión lectoría en inglés. Dada esta propuesta, la malla curricular renovada debería dar cuenta de estas intenciones." Jefe de carrera C.

"En el plan antiguo, como en el nuevo plan, producto de la renovación curricular, se consideran varias asignaturas que tributan a las competencia comunicativa, como lo expresé anteriormente." Jefe de carrera D.

"Estamos en rediseño pero es común para todas las pedagogías y no se ha tocado el tema" Jefe de carrera F. "Mas que una asignatura fijaría hitos de control durante el desarrollo del plan de estudio que es monitoreado y mejorado permanentemente." Jefe de carrera G.

De manera concluyente, solamente uno de los jefes de carrera, ha mencionado la posibilidad de considerar una renovación curricular; en tanto que el jefe de carrera $E$ ha valorizado que no se necesita algo adicional para mejorar las competencia comunicativa, pues en asignaturas sobre conocimiento pedagógico del contenido, las didácticas y aquellas donde se aprende a investigar en problemas de matemática ya está abordado. 
En otra apreciación se desestima una asignatura que aborde los temas, pese a la importancia concedida en la primera respuesta, defendiendo el establecimiento de hitos de control a través de un monitoreo, pero no explicita cómo se gestionaría. De forma general, los jefes de carrera hacen referencia a las habilidades comunicativa o a la competencia de la comunicación como eje transversal de sus programas de estudio. En la eventualidad de un rediseño curricular, lo someterían a evaluación y/o discusión, pero en definitiva no consideran que sea necesario integrar una asignatura que desarrolle estas habilidades. Para la mayoría ya existen las asignaturas que las desarrollan. Cabe mencionar que resulta interesante que se propongan hitos de control a lo largo de la formación del futuro profesor o futura profesora, pues daría cuenta de una evolución en el desarrollo de estas habilidades por parte de los futuros y futuras profesionales. De alguna manera se relacionaría con la preocupación de principios de siglo que mostraba las competencia comunicativa como algo no tratado (Rodríguez, 2000), de modo que se podría observar la evolución de ellas en el proceso formativo. Desde esta mirada, al tener cierto control en el proceso de formación no podría tomar por sorpresa a los titulados al momento de enfrentar el aula escolar (García, 2008) y, probablemente, se verían menos débiles en los aspectos comunicativos de su profesión (Gokalp, 2015).

En resumen, la encuesta a los jefes de carrera deja en evidencia que la preocupación por la competencia de la comunicación de la matemática queda en un plano transversal sin necesidad de corregirla o mejorarla de manera explícita. No obstante, sería apropiado examinar las percepciones del futuro profesor sobre su desarrollo profesional en esta materia. Una forma de pesquisar esta información podría ser a través de un diario de sus experiencias de práctica (Pop, 2015) con énfasis en lo comunicativo. Algunas acciones en pos de establecer este carácter mediador podrían darse en forma de un ciclo formativo específico en la formación inicial sobre la comunicación de la matemática, el cual debería estar configurado por un fuerte componente de matemática. Por ejemplo, que involucre la resolución de problemas de matemática. Esto se justifica en que en los momentos en que exista exposición de soluciones por parte de los estudiantes, sería el momento apropiado para llevarles a integrar aspectos de la competencia comunicativa que no se hayan desarrollado. En efecto, se busca provocar la reflexión previa a una clase con alumnos reales, considerando que las reflexiones del futuro Profesor se relacionan estrechamente con su práctica futura. (Miller y Baker, 2001). En otros aspectos, se debe desarrollar su capacidad argumentativa, el uso del léxico matemático apropiado, y el adecuado uso de diferentes representaciones de un objeto matemático acompañado de material manipulativo o recursos tecnológicos según sea el caso. Así, considerar también los aspectos sociales del aula en que el profesor debe identificar el tipo de discurso que utiliza, saber escuchar a sus alumnos, dar la oportunidad de participar en la clase entre otros (Saló, 2006).

\section{DISCUSIÓN FINAL}

De acuerdo a los resultados de la investigación se concluye que en el país aún se debe instalar el desarrollo de competencia comunicativa para enseñar matemática en futuros profesores en la formación inicial. Actualmente se considera que la competencia comunicativa del profesor de matemática está relacionada con elementos de la comunicación oral y escrita que debería desarrollar todo profesional. De acuerdo con las nuevas bases curriculares de 3ro. y 4to. medio en Chile, en los próximos años los profesores se verán llamados a revisar sus prácticas pedagógicas para cubrir los nuevos desafíos que plantean dichos lineamientos. Por ejemplo, asignaturas como Formación Ciudadana, constituye una oportunidad para que los estudiantes dialoguen acerca de matemática con profesores de otras asignaturas en pos de resolver temáticas de interés ciudadano. Así debe propiciarse antes en el aula de matemática, la posibilidad de que los escolares desarrollen la habilidad de usar la matemática en una variedad de situaciones y contextos (Niss, 2002). De este modo, el futuro profesor debe prepararse para "orquestar" en sus clases el tipo de discusiones que habitualmente no se llevan a cabo (Smith y Stein, 2011). Luego, de acuerdo con lo analizado en la investigación, existe en el futuro cercano una oportunidad para incorporar una nueva mirada en términos de las competencias comunicativas para el futuro profesor por los desafíos que plantea el país en materia de educación. Incluso se podría generar que se produzcan discusiones entre profesores acerca de los que sucede en el aula en el aspecto comunicativo (Arcavi, 2016) y que se visualicen oportunidades de formación continua.

Por consiguiente, al revisar el cómo se comunica el contenido matemático en el aula de educación media y, probablemente, cómo se comunican otras ciencias, se podría instalar como un nuevo tema de debate en formación inicial del profesor. Se trata de un diálogo que debe ir acompañado y complementado del trabajo en investigación y desarrollo de los principales referentes en esta materia, sin perder de vista que el profesor de matemática debe ser un mediador entre el conocimiento matemático y el alumnado (Planas, 2012). Se puede visualizar que con este reporte se puede iniciar la discusión en el país sobre un elemento que podría ser considerado como relevante en la formación inicial del profesor de matemática, puesto que no es un tema necesariamente de interés evidente al menos en estas instituciones consultadas. Sin duda, son muchos y diversos los elementos a considerar en la formación inicial del profesor del país. Bastante se ha avanzado en investigación en educación matemática en Chile, y las visiones de los investigadores chilenos podrían iluminar 
la importancia de esta y de otras competencias profesionales a desarrollar en el futuro profesor desde la voz de los expertos formados en el área (Pino et al, 2018).

A modo de resumen, el siguiente diagrama (Figura 1) muestra la relación entre los componentes a considerar en la formación inicial del profesor de matemática en Chile y la competencia comunicativa matemática. En el diagrama, el profesor de matemática en formación, en aspectos de comunicación de la matemática, está sujeto al programa de formación que ha escogido y que se encuentra respaldado por la Comisión Nacional de Acreditación, a las decisiones de los jefes de carrera respectivos, quienes representan la opinión experta, y a la realidad nacional en temas curriculares y educación escolar.

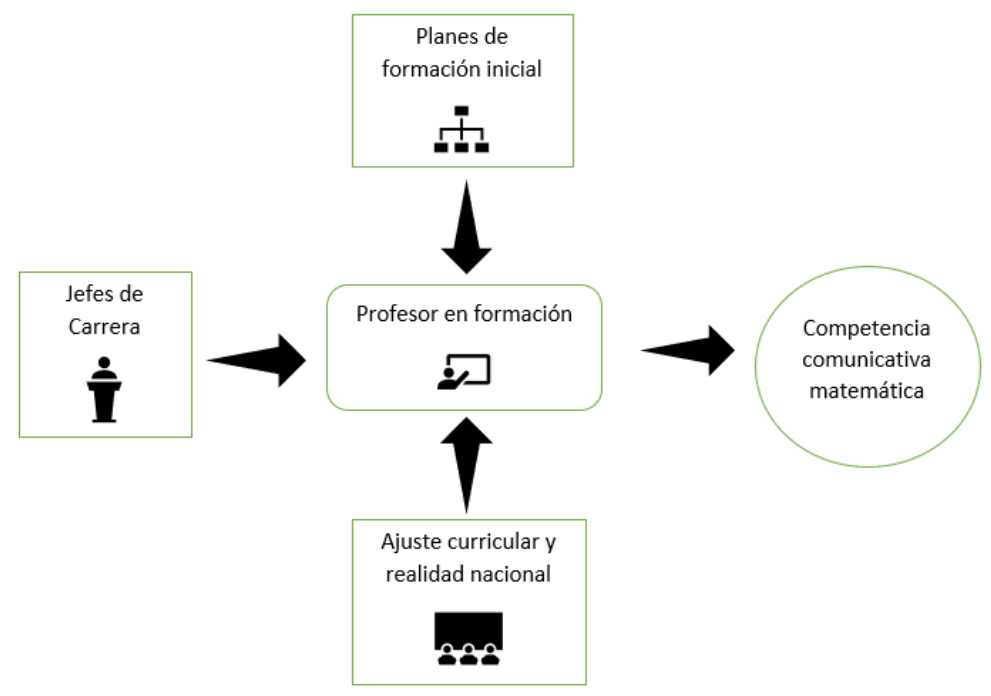

Fig. 1. Relación entre los componentes a considerar en la formación inicial del profesor de matemática en Chile y la competencia comunicativa matemática.

Podemos observar que el profesor se encuentra rodeado por los tres elementos antes mencionados. Sin embargo, se dirige de manera individual, de cierta manera, a la competencia comunicativa matemática, en el sentido que cuenta con herramientas para comunicarse profesionalmente y para el diseño de la enseñanza, pero no se evidencia, en la investigación realizada, el desarrollo de esta competencia.

\section{CONCLUSIONES}

De acuerdo al trabajo presentado y a los resultados obtenidos se pueden plantear las siguientes conclusiones principales:

1.- Los programas de formación inicial del profesor de matemática de las universidades del $\mathrm{CRUCH}$ contemplan, en sus planes de formación, la comunicación de la matemática, desde el punto de vista del diseño de la enseñanza, es decir, desde el ámbito profesional.

2.- La competencia de la comunicación de la matemática, en general, entre los jefes de carreras de pedagogía en matemática en Chile, queda en un plano transversal en la formación inicial del profesor de matemática.

3.- Los jefes de carrera estiman que la competencia comunicativa de la matemática es importante para el desarrollo profesional del docente. Sin embargo, proponen solo, en un eventual rediseño curricular, hitos de control más que una asignatura específica.

\section{AGRADECIMIENTOS}

Proyecto DICYT-USACH 31633VD Caracterización y Desarrollo de Competencia Comunicativa en la Formación de Profesores de Matemática, financiado por la Vicerrectoría de Investigación Desarrollo e Innovación y Proyecto "Proyecto de cooperación internacional de investigación. Innovación de la enseñanza y desarrollo curricular en la formación inicial docente" Financiado por DAAD., en Formación de Profesores financiado por Servicio Alemán de Intercambio Académico DAAD. 


\section{REFERENCIAS}

Arcavi, A., Promoviendo conversaciones entre docentes acerca de clases filmadas de matemáticas, Cuadernos de investigación y formación en educación matemática, 11(15), 385-396, Costa Rica (2016)

Carrión, C., M. Soler y M. Aymerch, Análisis de la validez de contenido de un cuestionario de evaluación del aprendizaje, doi: 10.4067/S0718-50062015000100003, Formación Universitaria, 8(1), 13-22 (2015)

Domingo, J., Percepción del profesorado sobre la competencia comunicativa en estudiantes de magisterio, Perfiles Educativos, XXXV, 142, 54-74 (2013)

Fernández, I. e I. Cuadrado, Competencia comunicativa del profesorado y desarrollo emocional del adolescente, International Journal of Developmental and Educational Psychology, ISSN: 0214-9877, INFAD Revista de Psicología, 2 , 299-308 (2010)

Gairín, J., Formación de Profesores basada en competencia, Bordón, 63(I), 93-108 (2011)

Galvis, R.V., De un perfil docente tradicional a un perfil docente basado en competencias, Acción pedagógica, 16, 48-57 (2007)

Giménez, J. y C. Vargas, Competencia comunicativa matemática y formación docente, En V. Font y V. Larios (Eds.) Competencia profesionales del profesor de matemática de secundaria, ISBN: 978-84-475-3558-3, Universitat de Barcelona, E-Book, Diciembre 2012, 103-114 (2012)

Gokalp, M., Investigating classroom teaching competencies of pre service elementary mathematics teachers, Eurasia Journal of Mathematics, Science and Technology Education, 12(3), 503-512 (2016)

Hernández, R., C. Fernández y P. Baptista, Metodología de la Investigación, 6aㅡ Ed., McGrawHill Education, Interamericana Editores, México (2014)

Ibañez, C., Un análisis crítico del modelo del triángulo pedagógico, Una propuesta alternativa, Revista Mexicana de Investigación Educativa, 12(32), enero-marzo, 435-456 (2007)

Leiblich, A., R. Tuval-Mashiachy y T. Zilber, Narrative research: Reading, analysis, and interpretation, Thousand Oaks, CA: Sage Publications (1998)

Lomas, C., Aprender a comunicar (se) en las aulas, Àgora Digital, 5, Grupo de Investigación Àgora de la Universidad de Huelva (2003)

Miller, K. y D. Baker, Mathematics and science as social practices, investigating primary student teacher respoonses to a critical epistemology, Ways of Knowing Journal, 1(1), 39-46 (2001)

Niss, M., Mathematical competencies and the learning of mathematics: The danish KOM project (Proyecto KOM), The national academies (2002)

Pavié, A., Formación Docente: hacia una definición del concepto de competencia profesional docente, Revista Electrónica Interuniversitaria de Formación del Profesorado, 14(1), 67-80 (2011)

Pimm, D., Speaking mathematically-Communication in mathematics classrooms, London, Routledge (1987)

Pino-Fan, L., I. Guzmán-Retamal, M. Larraín y C. Vargas-Díaz, La Formación inicial de profesores en Chile: 'Voces' de la comunidad chilena de investigación en educación matemática, Uniciencia, 32(1), 68-88 (2018)

Planas, N., Teoría, Crítica y Práctica de la Educación Matemática, Barcelona, Graó (2010)

Pop, R., Understanding pre-service trainees' perceptions of their teacher training experience, Procedia - Social and Behavioral Sciences, 209, 378-382 (2015)

Saló, N., Estrategias de comunicación en el aula, El diálogo y la comunicación interactiva, CEAC, Barcelona (2006)

Smith, M. y M.K. Stein, 5 practices for orchestrating productive mathematics discussions, Reston, Va., NCTM (2011)

Stemler, S., An overview of content analysis, Practical Assessment, Research \& Evaluation, 7(17) (2001)

Toledo, D. y D. Wittemberg, Reformulando la carrera docente en Chile, Evidencia internacional seleccionada, OECD (2015)

Vargas, C., Evolución de la competencia comunicativa matemática en un contexto de master de Formación de Profesores de matemática. La evolución de Ester, En Actas de Congreso de Educación Matemática de América Central y el Caribe, 6 al 8 de noviembre de 2013, Santo Domingo, República Dominicana (2013)

Villalta, M. y S. Martinic, Interacción didáctica y procesos cognitivos, Una aproximación desde la práctica y discurso del docente, Universitas Psychologica, 12(1), 221-233 (2013)

Villalta, M., Análisis de la conversación: una propuesta para el análisis de la interacción didáctica en la sala de clases, Estudios pedagógicos, XXXV(1), 221-238 (2009)

Villalta, M., S. Martinic y M. Guzmán, Elementos de la interacción didáctica en la sala de clase que contribuyen al aprendizaje en un contexto social vulnerable, ISSN: 14056666, RMIE, 2011, 16(51), 1137-1158 (2011) 
